\title{
Wnt signal activation induces midbrain specification through direct binding of the beta-catenin/TCF4 complex to the EN1 promoter in human pluripotent stem cells
}

\author{
Ji Young Kim¹,2, Jae Souk Lee ${ }^{1,2}$, Hyun Sub Hwang ${ }^{1}$, Dongjin R. Lee', Chul-Yong Park', Sung Jun Jung ${ }^{3}$,
} Young Rang You ${ }^{4}$, Dae-Sung $\mathrm{Kim}^{4}$ and Dong-Wook Kim,2

\begin{abstract}
The canonical Wnt signal pathway plays a pivotal role in anteroposterior patterning and midbrain specification during early neurogenesis. Activating Wnt signal has been a strategy for differentiating human pluripotent stem cells (PSCs) into midbrain dopaminergic (DA) neurons; however, the underlying molecular mechanism(s) of how the Wnt signal drives posterior fate remained unclear. In this study, we found that activating the canonical Wnt signal significantly upregulated the expression of EN1, a midbrain-specific marker, in a fibroblast growth factor signal-dependent manner in human PSC-derived neural precursor cells (NPCS). The EN1 promoter region contains a putative TCF4-binding site that directly interacts with the $\beta$-catenin/TCF complex upon Wnt signal activation. Once differentiated, NPCs treated with a Wnt signal agonist gave rise to functional midbrain neurons including glutamatergic, GABAergic, and DA neurons. Our results provide a potential molecular mechanism that underlies midbrain specification of human PSCderived NPCs by Wnt activation, as well as a differentiation paradigm for generating human midbrain neurons that may serve as a cellular platform for studying the ontogenesis of midbrain neurons and neurological diseases relevant to the midbrain.
\end{abstract}

\section{Introduction}

The midbrain is a portion of the central nervous system that develops between the diencephalon and the hindbrain and contains numerous relay centers for neural circuits in voluntary motor control (substantia nigra), reward response (ventral tegmental area), transmission of auditory and visual information (inferior and superior colliculi), and regulation of sleep-wake stages (reticular

\footnotetext{
Correspondence: D-S. Kim (sonnet10@korea.ac.kr) or D-W. Kim (dwkim2@yuhs.ac)

'Department of Physiology, Yonsei University College of Medicine, 50-1 Yonsei-ro Seodaemun-gu, Seoul 03722, Korea

${ }^{2}$ Brain Korea 21 PLUS Program for Medical Science, Yonsei University College of Medicine, 50-1 Yonsei-ro, Seodaemun-gu, Seoul 03722, Korea

Full list of author information is available at the end of the article

These authors contributed equally: Ji Young Kim, Jae Souk Lee.
}

formation $)^{1}$. Because multiple types of neurons reside in the dorsal, medial, and ventral domains of the midbrain, including glutamatergic, $\gamma$-amino butyric acid (GABA) ergic, and dopaminergic (DA) neurons ${ }^{2}$, malformation or functional impairment of midbrain neurons causes abnormal somatosensory information processing ${ }^{3,4}$, reward responses ${ }^{5}$, and sleep control ${ }^{6}$, as well as neurological disorders such as Parkinson's disease ${ }^{7}$. Given the important roles in normal brain function and the diversity, the mechanisms of development and differentiation of midbrain-specific neurons from stem cells have been of great interest for decades ${ }^{8}$.

Wnt signal is one of the key regulatory pathways that determine the regional identities of neural cells in developing embryos ${ }^{9,10}$, particularly those involved in 
specifying neural cells with posterior characteristics and implicated in midbrain morphogenesis ${ }^{11}$. Deleting Wnt1 resulted in the loss of the mid-hindbrain junction and failure in the midbrain and rostral hindbrain formation ${ }^{12-14}$. In contrast, inhibiting Wnt signal by endogenous inhibitors (Dickkopf homolog-1 [Dkk-1] and Cerberus) was shown to specify forebrain regions in vertebrates ${ }^{15,16}$. Moreover, Wnt ligands, depending on their concentration gradients, can either induce the expression of markers for posterior portions of the brain including engrailed 1 (En1), a midbrain marker ${ }^{17}$, and gastrulation brain homeodomain 2 (Gbx2), a hindbrain marker ${ }^{18}$, or suppress that of forebrain markers in explants of early avian embryos, suggesting that Wnt acts as a critical posteriorizing factor in vertebrates ${ }^{11}$. The hypothesis that similar developmental programs can be applied to the human system has been supported by recent differentiation studies using human pluripotent stem cells (PSCs) such as embryonic stem cells (ESCs) and induced pluripotent stem cells (iPSCs) in which the endogenous level of the Wnt signal determined the regional identity along the anteroposterior (AP) axis ${ }^{19}$; moreover, stimulating Wnt signal could facilitate the derivation of DA neurons that retain features of midbrain origin $^{20-22}$. It was known that DA neurons arise from the floor plate of ventral midbrain, not of hindbrain ${ }^{23}$. Thus, in attempts to induce authentic midbrain DA neurons, floor plate specification was first accomplished by early exposure of human PSCs to Sonic Hedgehog $(\mathrm{SHH})^{24}$. The differentiation strategy has been eventually evolved to combined modulation of both Shh and Wnt signals ${ }^{20}$. Despite all the implication of the Wnt signal in AP patterning and midbrain specification in both animal and human systems, the detailed molecular mechanism underlying how the Wnt signal drives posterior fate during neural differentiation and how inhibitors of glycogen synthase kinase 3 (GSK3), a key enzyme mediating the canonical Wnt signal, facilitate midbrain specification during human stem cell differentiation remained unclear.

Fibroblast growth factor 8 (FGF8) is secreted from the isthmic organizer located in the mid-hindbrain junction and thus composes a regulatory network for proliferation, survival, and patterning in the midbrain region ${ }^{25,26}$. It also acts as a key factor in inducing human PSCs into DA progenitors in cooperation with $\mathrm{SHH}$ because its expression in the developing embryonic brain is highly correlated with the region in which midbrain DA neurons arise $^{27}$. A previous study supported this idea by providing evidence that priming human ESC-derived neural precursor cells (NPCs) with FGF8 increased the number of cells with a midbrain fate in the culture ${ }^{28}$. As such, the molecular mechanism by which FGF8 contributes to fate determination or regional identity of developing neural cells in the midbrain area has been of interest in both developmental and stem cell biology.

Here we explored the role of canonical Wnt signal in AP patterning during neural differentiation of human PSCs. In particular, we investigated the molecular events during midbrain specification by Wnt activation while characterizing the interaction between the $\beta$-catenin/T-cell factor (TCF) complex and the EN1 promoter region and exploring the potential role of FGF8 in the same developmental context. Lastly, we examined the differentiation potential of NPCs with a midbrain fate via small molecule-based regulation of the canonical Wnt signal pathway.

\section{Materials and methods}

\section{Human PSC culture and differentiation}

We routinely cultured the undifferentiated human ESC line WA09 (H9, p37-49, WiCell Inc., Madison, WI, USA), retrovirus-derived iPSCs (iPS-WT3) ${ }^{29}$, and episomal vector-derived iPSCs (iPS-Epi3) ${ }^{30}$, and obtained the homogenous population of NPCs from them through modified dual SMAD inhibition and neural rosette selection as described previously ${ }^{31}$. The NPCs were used as a cellular platform to test the effects of Wnt signal on regional specification. Small molecules including 0.1-2 $\mu \mathrm{M}$ of 6-bromoindirubin-3'-oxime (BIO; Sigma-Aldrich, St. Louis, MO, USA), $10 \mu \mathrm{M}$ of 1-azakenpaullone (1-AKP; Sigma-Aldrich), and $5 \mathrm{mM}$ lithium chloride (LiCl; SigmaAldrich) were used to modulate Wnt signal. We used $100 \mathrm{ng} / \mathrm{ml}$ FGF8b (PeproTech, Rocky Hill, NJ, USA) as a control agent for midbrain specification ${ }^{28}$. To further differentiate NPCs into neurons, we mechanically detached the cell clumps and plated them after mild trituration on plates coated with Matrigel (Corning, Corning, NY, USA) and then cultured them in differentiation medium composed of DMEM/F12 supplemented with N2 (1×; Invitrogen, Carlsbad, CA, USA), B27 supplement (1×; Invitrogen), and various factors including $20 \mathrm{ng} / \mathrm{ml}$ glial cell line-derived neurotrophic factor, $20 \mathrm{ng} / \mathrm{ml}$ brainderived neurotrophic factor, $2 \mathrm{ng} / \mathrm{ml}$ transforming growth factor $\beta$ (TGF $\beta) 3$ (all from PeproTech), $200 \mu \mathrm{M}$ ascorbic acid, and $5 \mu \mathrm{M}$ forskolin (Sigma-Aldrich). In some experiments, we exposed NPCs to $200 \mathrm{ng} / \mathrm{ml}$ recombinant human SHH CIl24 (R\&D Systems, Minneapolis, MN, USA) and $100 \mathrm{ng} / \mathrm{ml}$ of FGF8b for a week before neuronal maturation.

\section{Semi-quantitative and quantitative (real-time) RT-PCR analyses}

We performed RT-PCR and quantitative RT-PCR (qRT-PCR) as described previously ${ }^{31}$; the primer sequences are listed in Supplementary Table 1. Briefly, we extracted total RNA using an Easy-Spin ${ }^{\bullet}$ Total RNA Purification Kit (iNtRON Biotechnology, Seoul, Korea), 
and then converted $1 \mu \mathrm{g}$ of RNA into cDNA with the iScript cDNA Synthesis Kit (Bio-Rad, Hercules, CA, USA). QRT-PCR was performed using SYBR ${ }^{\bullet}$ Premix ExTaq $^{\text {tix }}$ (TAKARA BIO Inc., Otsu, Japan) and the CFX96 Real-Time System (Bio-Rad). Beta-actin was used as an endogenous reference to calculate $\mathrm{Ct}$ values and relative expression levels $\left(2^{-\Delta \Delta C t}\right)$ of target genes. For semi-qRTPCR, we performed PCR with the $2 \times$ EmeraldAmp $^{\circledR}$ GT PCR Master Mix (TAKARA BIO Inc.) and 10 pmol of each primer (Supplementary Table 1). Amplified transcripts were normalized to the glyceraldehyde 3phosphate dehydrogenase (GAPDH)-specific signal.

\section{Lentiviral production for small hairpin RNA (shRNA)}

For the $\beta$-catenin knockdown experiment, we obtained two shRNA sequences from Addgene (Plasmid \#18803: pLKO.1 puro shRNA; \#19761: pLKO.1.sh.ß-catenin.1248; Cambridge, MA, USA). We constructed scrambled shRNA control using the HuSH ${ }^{\text {su }}$ shRNA plasmid pGFPC-shLent vector (OriGene, Rockville, MD, USA) and produced the viruses by transient transfection of 293T cells with lentiviral packaging plasmids (pLP1, pLP2, and pLP/VSVG; Invitrogen). We determined virus concentration using a Lenti-X Concentrator (Clontech, Mountain View, CA, USA) following the manufacturer's protocol, and we monitored knockdown efficiency by qRT-PCR and immunoblotting against $\beta$-catenin (see below).

\section{Immunocytochemistry}

Cells were fixed with $4 \%$ paraformaldehyde in phosphate-buffered saline (PBS) for $30 \mathrm{~min}$, washed with PBS, and perforated with PBS containing $0.1 \%$ Triton X100 for $10 \mathrm{~min}$. Then, the samples were incubated with blocking buffer (PBS containing 2\% bovine serum albumin) for $1 \mathrm{~h}$. The cells were incubated with primary antibodies diluted in blocking buffer at $4{ }^{\circ} \mathrm{C}$ overnight. The following primary antibodies were used: rabbit antiNestin (1:1000, Millipore, Billerica, MA, USA), mouse anti- $\beta$-catenin (1:1000, Santa Cruz Biotechnology, Dallas, TX, USA), goat anti-SOX1 (1:100, R\&D Systems), mouse anti-EN1 (1:50, DSHB, Iowa City, IA, USA), rabbit antiTUJ1 (1:1000, Covance, Richmond, CA, USA), goat antiOTX2 (1:300, R\&D systems), sheep anti-TH (1:300, PelFreez, Rogers, AR, USA), rabbit anti-TH (1:300, PelFreez), rabbit anti-glutamate (1:200, Sigma-Aldrich), rabbit anti-GABA (1:2500, Sigma-Aldrich), goat antiLMX1A (1:200, Santa Cruz), rabbit anti-FOXA2 (1:300, Millipore), mouse anti-synaptophysin (1:1000, Millipore), and rabbit anti-MAP2 (1:500, Millipore). The samples were washed three times with PBS and then incubated with fluorescence-tagged secondary antibodies (Alexa Fluor 350, Alexa Fluor 488, and Alexa Fluor 594, 1:500, Invitrogen) in blocking buffer for $30 \mathrm{~min}$ at room temperature. The cover slips were rinsed three times with PBS and mounted onto slides using mounting medium (Vector Laboratories, Burlingame, CA, USA). All images were obtained using a fluorescence microscope (DP71, FSX100; Olympus, Tokyo, Japan) or an LSM700 confocal microscope (Carl Zeiss, Oberkochen, Baden-Württemberg, Germany).

\section{Immunoblotting}

Cells were washed once with ice-chilled PBS and lysed in a RIPA buffer (Sigma-Aldrich) with a protease inhibitor cocktail (Roche Applied Science, Mannheim, BadenWürttemberg, Germany). Protein was quantified using the Bradford protein solution (Bio-Rad), and then $20 \mu \mathrm{g}$ of protein samples were separated on a $10 \%$ sodium dodecyl sulfate-polyacrylamide gel electrophoresis (SDS-PAGE) and transferred to a PVDF membrane (Millipore). After incubation with $5 \%$ non-fat milk in Tris-buffered saline containing $0.1 \%$ Tween 20 (TBS- $\mathrm{T}$ ) for $1 \mathrm{~h}$ at room temperature, the membrane was incubated with primary antibodies (mouse anti- $\beta$-catenin (Santa Cruz Biotechnology), mouse anti-EN1 (Abcam, Cambridge, UK), and mouse anti- $\beta$-actin (Sigma-Aldrich)) for $1 \mathrm{~h}$ at room temperature or overnight at $4{ }^{\circ} \mathrm{C}$. The membrane was washed three times with TBS-T and then incubated in a 1:3000 dilution of horseradish peroxidase-conjugated anti-mouse secondary antibody for $30 \mathrm{~min}$ at room temperature. The membranes were washed with TBS-T three times and developed with the enhanced chemiluminescence system (Pierce, Rockford, IL, USA) according to the manufacturer's protocols.

\section{Co-immunoprecipitation (Co-IP) assay}

Total cell lysates were prepared by NETN lysis buffer (100 mM NaCl, $20 \mathrm{mM}$ Tris-Cl, $0.5 \mathrm{mM}$ EDTA, and 0.5\% (v/v) NP-40) with a protease inhibitor cocktail (Roche Applied Science). Total protein was obtained from cell lysates by centrifugation at $12,000 \times g$ for $30 \mathrm{~min}$ at $4{ }^{\circ} \mathrm{C}$ and quantified using the Bradford protein solution (BioRad). The protein samples were incubated with a mouse anti-TCF-4 antibody (Upstate, Billerica, MA, USA) overnight at $4{ }^{\circ} \mathrm{C}$ and then incubated with protein GSepharose beads (GE Healthcare Bio-Sciences, Pittsburgh, PA, USA) for $1 \mathrm{~h}$ at $4{ }^{\circ} \mathrm{C}$ with rotation. The beads were collected by centrifugation, washed three times with the same lysis buffer, dissolved in sample buffer, and analyzed by SDS-PAGE followed by detection with mouse anti- $\beta$-catenin antibody (Santa Cruz Biotechnology).

\section{Chromatin immunoprecipitation (ChIP) assay}

For ChIP assays, cells were cultured with BIO for 3 days, at which point the cells were fixed in formaldehyde for 10 min, and then $0.125 \mathrm{M}$ glycine was added to stop the reaction. The cells were washed twice with PBS. The ChIP 
assays were performed with a ChIP kit (R\&D Systems) according to the manufacturer's instructions. Briefly, the fixed cells were lysed and sonicated to shear the genomic DNA into 200-500 bp fragments. The cross-linked complexes (DNA-protein) were immunoprecipitated with anti-TCF4 antibody (Millipore) or normal goat IgG (Santa Cruz). ChIP-enriched DNA was quantified by qRT-PCR with primer pairs designed to flank the putative TCFbinding element. The primer sequences are listed in Supplementary Table 2.

\section{Plasmid and reporter gene constructs}

Expression vectors for Flag-tagged constitutively active $\beta$-catenin ( $\beta$-catenin 4A; S33A, S37A, T41A, and S45A) and Myc-tagged wild-type TCF-4 were obtained from Addgene (plasmids \#24204 and \#16514). The human EN1 promoter fragment $(-4961 \sim-81)$ was amplified by PCR using ExTaq (TAKARA BIO Inc.) and human dermal fibroblast's genomic DNA as a template, and was subcloned into the pGL3-basic Luciferase Reporter Vector (Invitrogen). Cloning was performed using an In-fusion HD Cloning Kit (Clontech Laboratories Inc., Mountain View, CA, USA). The primers were generated using SnapGene software and are listed in Supplementary Table 3. The sequence of the EN1 promoter was aligned with the sequence of human EN1 (engrailed homeobox 1) on chromosome 2 (NCBI, NG_007123).

\section{Transfection and luciferase reporter assays}

For reporter assays, HEK 293 cells or NPCs were seeded into a 12-well culture plate and transiently transfected with $0.1 \mu \mathrm{g}$ reporter plasmid and $30 \mathrm{ng}$ pRL-SV40 plasmid (Promega, Madison, WI, USA) containing the Renilla luciferase gene under the control of the Simian virus 40 promoter using polyethyleneimine (PEI, Sigma-Aldrich). For the over-expression of $\beta$-catenin and TCF4, cells were transfected with plasmid using PEI. Forty-eight hours after transfection, the cells were harvested, and luciferase activity was measured and normalized to Renilla luciferase activity. The amount of DNA in each transfection was kept constant by adding an appropriate amount of empty vector.

\section{Electrophysiological recording}

Electrophysiological recording of the differentiated neurons was conducted by whole-cell patch-clamp technique with an EPC 10 USB amplifier (HEKA Electronik, Lamprecht, Germany). The differentiated neurons attached on the glass cover slips were transferred to a 0.5$\mathrm{ml}$ recording chamber that was superfused continuously with a normal Tyrode solution by gravity at a rate of approximately $10 \mathrm{ml} / \mathrm{min}$. The recording electrodes were manufactured from borosilicate glass capillaries (Sutter Instrument Company, Novato, CA, USA) using a PC-10 puller (Narishige Co., Tokyo, Japan), and the resistance of the pipettes was 4-6M $\mathrm{M}$ when filled with the pipette solution described below. The cell membrane capacitance and series resistance were electronically compensated (typically $80 \%$ ). After a giga-seal was obtained by negative suction, the cell membrane was ruptured by gentle suction to establish a whole-cell configuration. Data were filtered at $3 \mathrm{kHz}$, digitized at $10 \mathrm{kHz}$, and analyzed using Pulse ver. 8.67 (HEKA Electronik) and Origin 6.1 software (MicroCal, Northampton, MA, USA). All experiments were performed at room temperature $\left(21-24^{\circ} \mathrm{C}\right)$. The membrane potentials and currents were recorded with the following normal Tyrode solution: $143 \mathrm{mM} \mathrm{NaCl}, 5.4 \mathrm{mM}$ $\mathrm{KCl}, 0.5 \mathrm{mM} \mathrm{MgCl}_{2}, 1.8 \mathrm{mM} \mathrm{CaCl}_{2}, 0.5 \mathrm{mM} \mathrm{NaHPO}_{4}, 10$ $\mathrm{mM}$ glucose, and $5 \mathrm{mM}$ HEPES. The $\mathrm{pH}$ level was adjusted to 7.4 with $\mathrm{NaOH}$. The pipette solution contained $150 \mathrm{mM} \mathrm{KCl}, 1.0 \mathrm{mM} \mathrm{MgCl}_{2}, 10 \mathrm{mM}$ HEPES, 5 mM EGTA, and $2 \mathrm{mM} \mathrm{Mg-ATP,} \mathrm{and} \mathrm{the} \mathrm{pH} \mathrm{level} \mathrm{was}$ adjusted to 7.2 with $\mathrm{NaOH}$.

\section{Statistics}

Values are expressed as the mean \pm S.E.M unless indicated otherwise. We used ANOVA and Student's $t$ test using SPSS software version 12.0 to determine the statistical significance.

\section{Results}

Activating the canonical Wnt pathway induces midbrainspecific gene expression in human PSC-NPCs

To obtain a cellular platform for investigating the role of Wnt signal in AP patterning, we generated a NPC population from human PSCs through the simultaneous inhibition of the bone morphogenetic protein and TGF $\beta$ signals with small molecules (dorsomorphin and SB431542) ${ }^{31}$. We observed extensive neural rosette formation in the center of the colonies 4-5 days after attachment of embryoid bodies that were exposed to dorsomorphin and SB431542 for 4 days (Fig. 1a). Mechanically isolated neural rosettes were highly positive for SOX1 and Nestin and maintained the typical morphology of the neural rosette during expansion (Fig. 1b, c). Because neural rosettes are an NPC population that can respond to extrinsic patterning cues directing regional specification $^{32,33}$, we used these cells to investigate cellular events during AP patterning by modulating Wnt signal pathway.

To test the potential effect of Wnt signal activation on AP patterning, we first treated human ESC-derived NPCs with a small-molecule GSK3 inhibitor, BIO, which is known to activate the canonical Wnt signal pathway ${ }^{34,35}$, and then examined the expression of the following representative AP markers by qRT-PCR: brain factor 1 (BF1, a forebrain marker ${ }^{36}$ also known as FOXG1), EN1, and $G B X 2$. As a positive control culture condition, we 


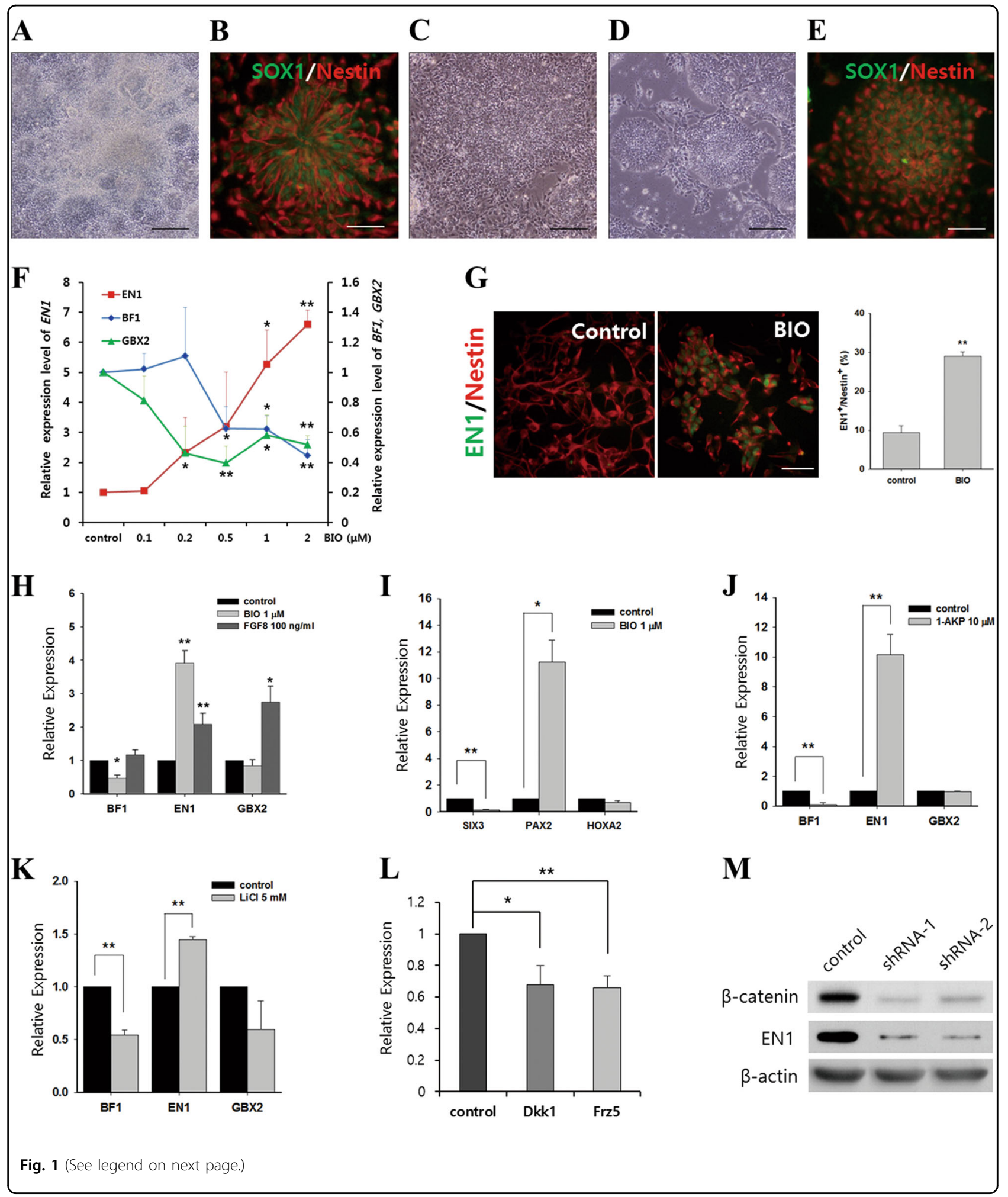

treated some cells with $100 \mathrm{ng} / \mathrm{ml} \mathrm{FGF8} \mathrm{because} \mathrm{it} \mathrm{was}$ shown to induce midbrain-fated NPCs when treated at early stage of $\mathrm{NPCs}^{28}$. Once treated with BIO, NPCs proliferated and formed compact colonies while maintaining the expression of the NPC markers (Fig. 1d, e). Quantitative gene expression analysis following seven days of treatment revealed that EN1 expression increased gradually in a dose-dependent manner up to five times at 
(see figure on previous page)

Fig. 1 Activation of Wnt signal induces midbrain characteristics in human ESC-derived NPCs. a Efficient induction of neural rosette cells from human ESCs by co-treatment with dorsomorphin and SB431542. b Strong immunoreactivity for SOX 1 and Nestin in neural rosette cells. Morphology of neural rosette cells expanding in either the absence (c) or the presence of $1 \mu \mathrm{M} \mathrm{BIO} \mathrm{(d).} \mathrm{e} \mathrm{NPCs} \mathrm{treated} \mathrm{with} \mathrm{BIO} \mathrm{maintained} \mathrm{immunoreactivity} \mathrm{for}$ SOX1 and Nestin. f Treatment with BIO upregulated EN1 expression and downregulated expressions of BF1 and GBX2 in dose-dependent manner. $\mathbf{g}$ $\mathrm{BIO}$ treatment significantly increased the number of EN1-positive neural cells. $\mathbf{h}$ The inductive effect of BIO treatment on midbrain fate appeared to be more specific than that of FGF8. i Expression pattern of another set of regional markers (SIX3 for forebrain; PAX2 for midbrain; and HOXA2 for hindbrain) supported the midbrain-biased fate of NPCs treated with BIO. Treatment with other known GSK3 inhibitors, 1-AKP (j) and LiCl (k), resulted in regionalization comparable to BIO treatment. I Treating NPCs with Wnt antagonists ( $100 \mathrm{ng} / \mathrm{ml} \mathrm{DKK-1}$ and $500 \mathrm{ng} / \mathrm{ml}$ frizzled-5) downregulated the endogenous level of EN1 transcript in NPCs. $\mathbf{m}$ Immunoblot for $\beta$-catenin and EN1 protein after introduction of two different $\beta$-catenin-specific shRNAs (shRNA-1 and shRNA-2). EN1 protein level was directly downregulated by $\beta$-catenin knockdown. $\beta$-actin was a loading control. All data are expressed as mean \pm S.E.M. Statistical significance was estimated using Student's $t$ test $(\mathbf{g}, \mathbf{i}, \mathbf{j}$, and $\mathbf{k})$ or one-way ANOVA $(\mathbf{f}, \mathbf{h}$, and $\mathbf{I})$ from at least three independent experiments; ${ }^{*} p<0.05,{ }^{* *} p<0.01$. Scale bar: $200 \mu \mathrm{m}$ for $\mathbf{a}, \mathbf{c}$, and $\mathbf{d} ; 50 \mu \mathrm{m}$ for $\mathbf{b}, \mathbf{e}$, and $\mathbf{g}$

$1 \mu \mathrm{M}$, whereas the $B F 1$ and $G B X 2$ expression decreased as the BIO concentration increased (Fig. 1f). Although the EN1 expression was gradually upregulated as the BIO concentration increased, detrimental effects on cell survival restricted us to use more than $2 \mu \mathrm{M}$ BIO for subsequent experiments (data not shown). Immunofluorescence staining supported the qRT-PCR results by showing an evident increase in the number EN1-positive cells in the BIO-treated group compared with the non-treated control (Fig. 1g). Interestingly, BIO treatment appeared to be more specific for inducing the midbrain marker than FGF8 treatment. The treatment with $1 \mu \mathrm{M}$ BIO increased EN1 expression by two folds compared to $100 \mathrm{ng} / \mathrm{ml}$ FGF8 treatment which instead increased the level of $G B X 2$ transcripts with no change of $B F 1$ expression (Fig. 1h). Upregulation of both EN1 and $G B X 2$ by FGF8 treatment might result from the fact that FGF8 affects the specification of both the midbrain and hindbrain $^{25}$. It was also intriguing that $B F 1$ expression was not affected by FGF8 treatment contradicting to the previous result ${ }^{28}$ (Fig. 1h). The presence of SOX1-positive cells in the neural rosettes (Fig. 1b) and the previous finding that FGF8 suppresses the forebrain fate only in the "primitive" NPCs (paired box protein 6 [PAX6]-positive, but SOX1-negative) ${ }^{28}$, indicate that a substantial number of the NPCs in our experiment are likely in the "definitive" (i.e., PAX6/SOX1-double positive) stage and thus less responsive to the activity of FGF8 in suppressing the forebrain fate. Nonetheless, BIO treatment potently increased $E N 1$ expression. This result suggests that $\mathrm{BIO}$ is a strong regionalizing cue and that our system stands valid to examine the effect of such regionalizing cue and its molecular mechanism.

Additional quantitative gene expression analysis with other AP markers, such as SIX homeobox 3 (SIX3; forebrain $\left.^{37}\right)$, paired box gene 2 (PAX2; midbrain $\left.^{38}\right)$, and homeobox protein HOXA2 (hindbrain ${ }^{39}$ ), yielded similar results (Fig. 1i), supporting the midbrain-specific regionalization of human ESC-derived NPCs following BIO treatment. We also observed the same AP marker expression patterns after BIO treatment on both NPCs derived from two iPSC lines (iPS-WT3 and Epi3; Supplementary Fig. 1A-B and data not shown) and human ESC-derived NPCs cultured in suspension as described previously $^{31}$ (Supplementary Fig. 1C), suggesting the robust effect of $\mathrm{BIO}$ on midbrain specification regardless of human PSC type or cultivation method.

To confirm whether the effect of BIO on midbrain specification was indeed through the activation of canonical Wnt signal, we tested other small molecules that inhibit GSK3 in different modes of action, such as 1$\mathrm{AKP}^{40}$ and $\mathrm{LiCl}^{41,42}$. Exposing human ESC-derived NPCs to 1-AKP robustly increased EN1 expression, whereas $B F 1$ expression level strongly decreased (Fig. 1j). LiCl treatment elicited similar gene expression patterns, although the fold changes in gene expression were lower than those of the other inhibitors (Fig. 1k). These data support that midbrain-specific gene expression results from the activation of canonical Wnt signal via GSK3 inhibition ${ }^{43,44}$.

As another approach to investigate the involvement of the canonical Wnt signal pathway in the midbrain specification, we inhibited endogenous Wnt signal in the NPC culture and examined the EN1 expression level. When the NPCs were cultured in the presence of Wnt signal antagonists such as DKK-1 or frizzled5-Fc (FZD-5), basal EN1 expression was found to be significantly lower in both cases (Fig. 11); significant diminution of EN1 expression by FZD-5 was also found in iPSC-derived NPCs (Supplementary Fig. 2). Furthermore, knockdown of $\beta$-catenin by transducing NPCs with specific shRNAs downregulated EN1 protein level as well as $\beta$-catenin (Fig. $1 \mathrm{~m}$ ). Taken together, our results strongly suggest that the canonical Wnt signal pathway directly contributes to the expression of midbrain-specific genes in human PSC-derived NPCs.

\section{The $\beta$-catenin/TCF complex binds directly to the EN1 promoter region}

Previous genetic studies in Xenopus and mice provided evidence that ablation of Wnt1 disrupts the AP axis, and that Wnt1 regulates EN1 expression in the earliest stages $^{12,45-47}$. These findings prompted us to ask whether 
A

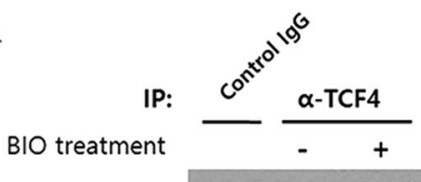

IB: $\alpha-\beta$-catenin

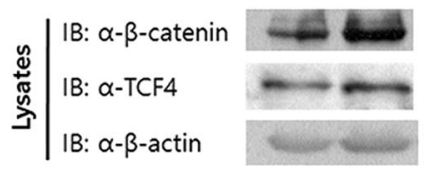

C

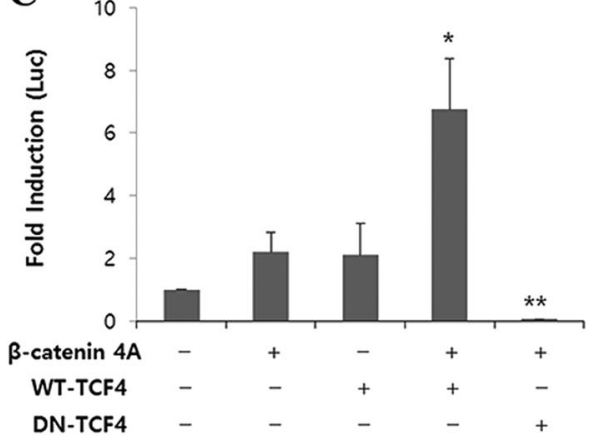

B

B-catenin

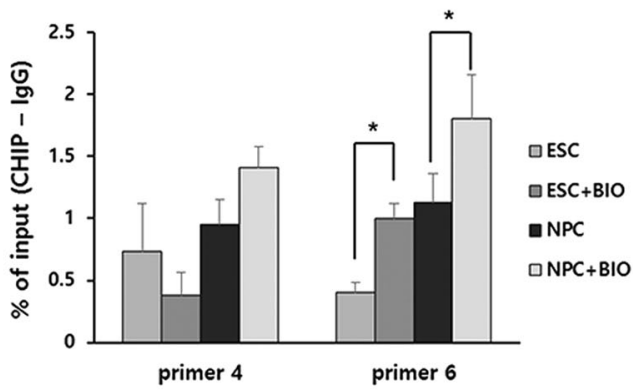

D

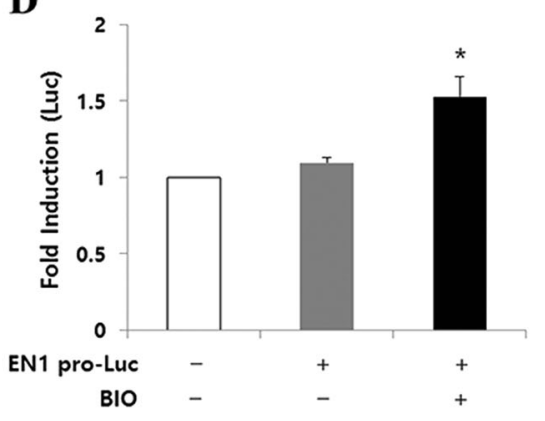

Fig. 2 EN1 expression is regulated by the $\beta$-catenin/TCF complex through direct binding to the EN1 promoter region. a Co-IP analysis showed that the interaction between $\beta$-catenin and TCF4 increased by BIO-treatment. $\mathbf{b}, \mathbf{c}$ ChIP assays for $\beta$-catenin/TCF4 complex to the putative binding motif of EN1 promoter. The $y$-axis represents the means \pm S.E.M. of the relative binding level of $\beta$-catenin (via TCF4) to each target sequence. c Expression of luciferase driven by a 5-kb-long regulatory region of human EN1 gene in HEK 293 cells. A reporter gene was strongly induced by overexpression of both constitutively active mutant of $\beta$-catenin (Cat4A) and WT-TCF4. $\mathbf{d}$ BIO treatment directly increased luciferase expression under the control of the EN1 regulatory sequence in human ESC-derived NPCs. Statistical significance was estimated using one-way ANOVA with multiple comparisons among groups from at least three independent experiments; ${ }^{*} p<0.05,{ }^{* *} p<0.01$.

EN1 expression is directly regulated through binding of transcriptional modulators, downstream of the canonical Wnt signal, to its regulatory DNA elements in the human system. First, we verified that the interaction between $\beta$ catenin and TCF4, a well-known interacting partner of nuclear $\beta$-catenin, was increased by BIO treatment in human ESC-derived NPCs on Co-IP assay (Fig. 2a)

We next sought to determine the regulatory element around the EN1 gene locus for the $\beta$-catenin/TCF complex binding. Because $\beta$-catenin lacks DNA-binding motifs, we searched putative binding sites for TCF4 using Patch 1.0 Transfac ${ }^{\circledR}$. We identified eight candidates localized within a $5-\mathrm{kb}$ region upstream of the human EN1 transcription start site (Supplementary Fig. 3A, sites $1-8$ ), but only one (site 6) out of the eight putative binding motifs was confirmed to be the consensus sequence for TCF4 (Supplementary Fig. 3B). To determine whether the $\beta$-catenin/TCF4 complex binds to the putative binding motif in response to the activation of the Wnt signal, we performed a ChIP assay using an antibody specific for $\beta$-catenin after treatment with BIO. In result, the $\beta$-catenin/TCF4 complex binding to site 6 was markedly induced after BIO treatment in human ESCderived NPCs, whereas binding to other sites with nonconsensus sequences (site 4, for example) did not take place significantly (Fig. 2b). Interestingly, $\beta$-catenin/TCF4 complex binding to site 6 also increased markedly with BIO treatment in human ESCs (Fig. 2b). Our result demonstrates that $\beta$-catenin/TCF4 complex binding not only appears to increase with neural differentiation (ESC vs. NPC in Fig. 2b), but also increases in response to Wnt activation (NPC vs. NPC + BIO in Fig. 2b). These results suggest that the accessibility of regulatory elements situated in $E N 1$ gene depends on developmental stage as well as the recruitment of $\beta$-catenin/TCF4 complex to its regulatory element via Wnt activation.

In order to further examine whether the $\beta$-catenin/ TCF4 complex directly induces EN1 transcription, we performed the promoter activity assay using an EN1 promoter-luciferase reporter plasmid in HEK 293 cells. A constitutively active mutant of $\beta$-catenin (Cat4A), wildtype (WT)-TCF4, and dominant-negative (DN)-TCF4 expression plasmid were transfected either alone or in combination and then the luciferase activity was 
measured. Surprisingly, co-transfection of the Cat $4 \mathrm{~A}$ and WT-TCF4 dramatically increased transcriptional activity compared with transfection of the Cat4A or WT-TCF4 alone (Fig. 2c). In addition, we observed no transcriptional activity when we co-introduced Cat4A with DN-TCF4 (Fig. 2c). These findings indicate that $\beta$-catenin and TCF4 bind to the EN1 promoter and induce the transcription as a complex. Finally, when the EN1 promoter activity was tested in human ESC-derived NPCs, BIO treatment indeed slightly but significantly upregulated luciferase activity compared with the non-treated control group (Fig. 2d). Collectively, our data demonstrate that activating the canonical Wnt pathway induces EN1 expression through direct binding of $\beta$-catenin/TCF to the EN1 promoter region in human ESC-derived NPCs.

\section{FGF signal is required for the induction of EN1 by Wnt signal activation}

Previous evidence demonstrating the involvement of FGF signal in AP patterning and in inducing midbrain and rostral hindbrain cells ${ }^{9,26,46-49}$ prompted us to further investigate the role of FGF signal in midbrain specification by Wnt activation. As shown in Fig. 3a, we detected the endogenous expression of various FGF transcripts in the NPC culture. We also found that treatment of GSK3 inhibitor increased the expression of FGF8 as well as functionally related FGFs (FGF17, 18) (Fig. 3a), which is consistent with the previous findings in rodents that FGF8 expression is induced by the activation of the Wnt signal in the midbrain ${ }^{9,46,47}$. To determine whether an endogenous FGF signal is required for basal EN1 expression, we treated human ESC-derived NPCs with $2.5 \mu \mathrm{M}$ SU5402, an FGF receptor tyrosine kinase inhibitor, for 5 days. Gene expression analysis showed that blocking endogenous FGF signal significantly reduced EN1 expression (Fig. 3b), indicating that endogenous FGF signal is at least partially responsible for the basal expression of $E N 1$. To further examine the involvement of FGF signal in induction of EN1 expression by Wnt activation, we treated NPCs with either recombinant FGF8 or SU5402 in the presence of BIO. After 5 days of treatment, qRT-PCR analysis revealed that co-treatment with FGF8 and $\mathrm{BIO}$ upregulated EN1 expression more than treatment with BIO alone, but the increment did not reach statistical significance (Fig. 3c). However, when FGF signal is blocked by treatment with SU5402 in the presence of $\mathrm{BIO}$, the increased EN1 expression, attributed to endogenous plus BIO-induced FGFs, was abrogated (Fig. 3c). Immunoblot analysis showed a similar pattern of EN1 protein level to the results from qRT-PCR (Fig. 3d, e). Intriguingly, the total protein levels of $\beta$-catenin increased significantly in all BIO-treated groups compared with the untreated control but were unaffected by the presence of FGF8 or SU5402 (Fig. 3d, e). A recent study showed that
FGF receptor tyrosine kinase directly phosphorylates $\beta$ catenin at Tyr142 upon FGF binding, liberating $\beta$-catenin from the membrane and allowing for transcriptional activation in the nucleus ${ }^{50}$. This result prompted us to examine whether FGF signal would influence on the subcellular localization of $\beta$-catenin under the active Wnt signal. Immunocytochemical analysis of $\beta$-catenin showed that $\beta$-catenin immunoreactivity was localized in the plasma membrane in the control group, but BIO treatment remarkably localized $\beta$-catenin in the nuclei (Fig. 3f, g). Moreover, FGF signal was unlikely to affect its translocation to the nucleus by Wnt activation (the BIO group vs. the $\mathrm{BIO}+\mathrm{FGF} 8$ or $\mathrm{BIO}+\mathrm{SU}$ groups in Fig. $3 \mathrm{f}, \mathrm{g}$ ). Taken together, our data suggest that FGF signal positively influences EN1 expression in human ESC-derived NPCs but in a manner independent of the nuclear translocation of $\beta$-catenin.

\section{Differentiation potential of midbrain NPCs induced by BIO treatment}

Next, we explored the differentiation potential of the midbrain-fated NPCs. After 1 week of BIO treatment, we differentiated the NPCs under neuronal maturation conditions (see Materials and Methods). After 3 weeks of neuronal differentiation, immunocytochemical analysis showed that $62.3 \pm 3.4 \%$ of the total cells were positive for TUJ1 in the BIO-treated group, whereas $33.9 \pm 6.1 \%$ of the cells turned positive for TUJ1 in the control group (Fig. 4a), possibly attributed to Wnt promoted neurogenesis $^{51}$. As we expected, BIO-treated NPCs generated more TUJ1-positive neuronal cells that co-expressed EN1 $(67.6 \pm 6.6 \%)$ than the control group $(10.6 \pm 12.7 \%, p<$ 0.05; Fig. 4b). Notably, the majority of EN1-positive cells in BIO-treated group was also positive for orthodenticle homeobox 2 (OTX2) (70.6 $\pm 10.0 \%$; Fig. 4b). Given that dual immunoreactivity of EN1 and OTX2 is indicative of midbrain fate, ${ }^{11,21}$ the majority of neurons expressing EN1 was indeed of midbrain character. Interestingly, glutamate (Glu)-positive putative excitatory neurons were the majority $(58.7 \pm 6.7 \%)$ among EN1-positive cells followed by GABA-positive $(16.7 \pm 5.3 \%)$ and $\mathrm{TH}$-positive cells $(10.2 \pm 2.8 \%)$ (Fig. 4c). Considering the fact that glutamatergic excitatory and GABAergic inhibitory neurons mainly arise within the dorso-medial region whereas DA neurons are born in the most ventral region in the midbrain $^{52}$, BIO treatment appeared to drive NPCs toward dorso-medial midbrain fates. To further explore whether the NPCs treated with BIO could also potentially generate neurons of the ventral midbrain (e.g., DA neurons), we treated NPCs with $200 \mathrm{ng} / \mathrm{ml} \mathrm{SHH}$ and $100 \mathrm{ng} / \mathrm{ml} \mathrm{FGF8}$ for a week following $\mathrm{BIO}$ treatment. Immunostaining followed by quantitative analysis revealed that the treatment with SHH and FGF8 increased the proportion of TH-positive cells among EN1-positive cells $(20.9 \pm 2.8 \%)$ 
A

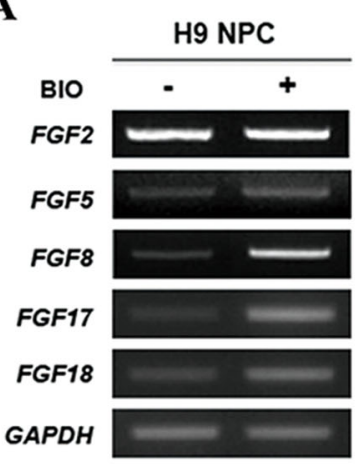

B

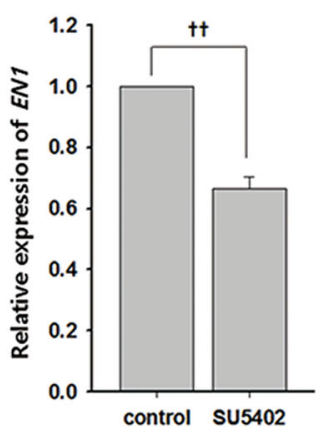

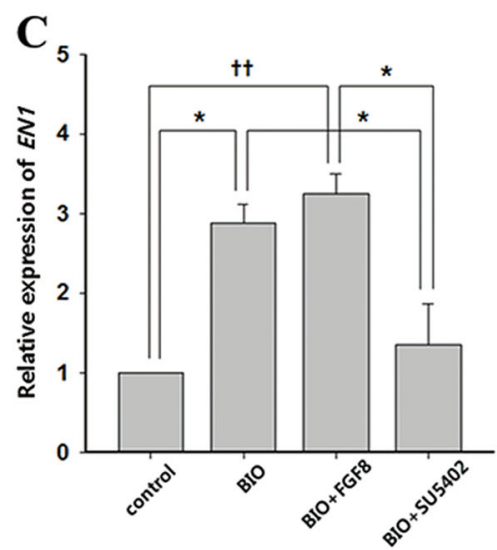

E
D

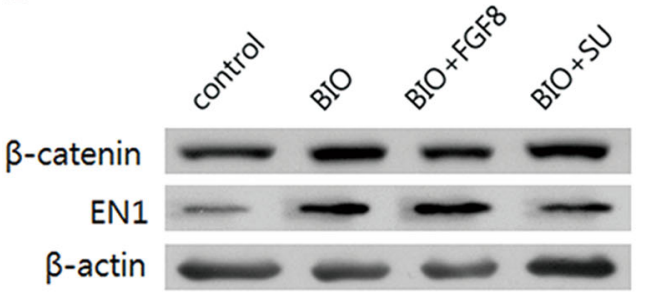

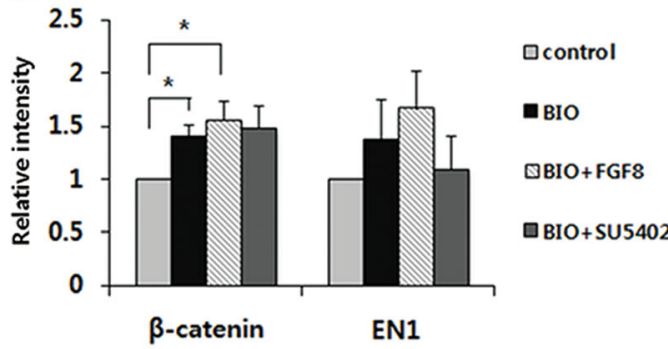

G
F $\beta$-catenin/DAPI
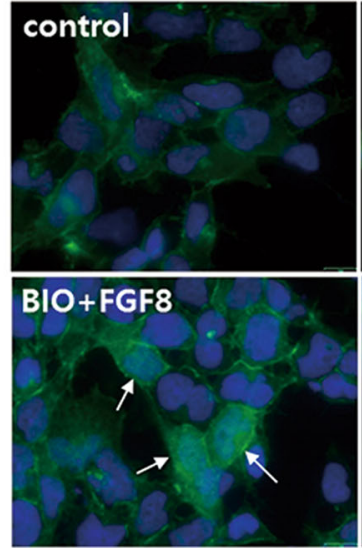

BIO
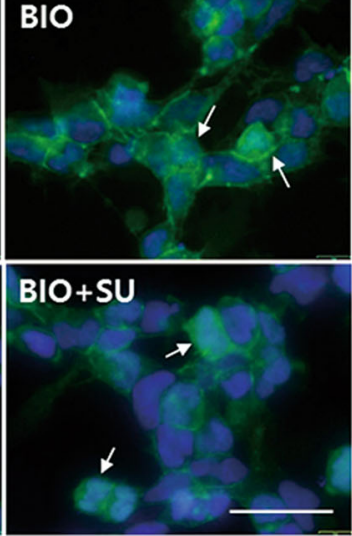

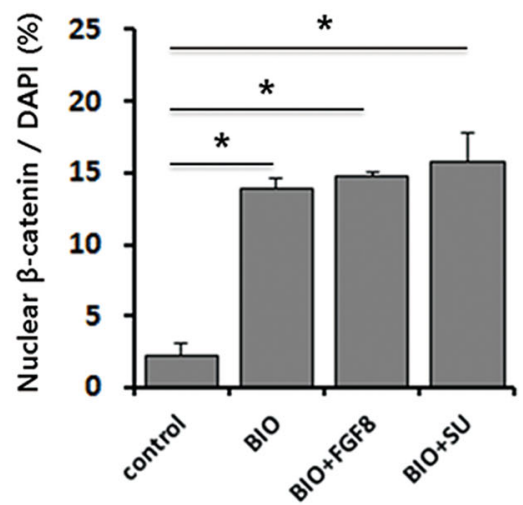

Fig. 3 FGF signal is required for induction of EN1 by activation of Wnt signal. a Semi-qRT-PCR analysis showed endogenous and BIO-induced expression of various FGFs in human ESC-derived NPCs. b Inhibition of endogenous FGF signals by SU5402 significantly decreased basal EN1 expression. c Co-treatment with BIO and FGF8 slightly increased EN1 expression more than BIO alone whereas co-treatment of SU5402 with BIO abrogated the upregulation of EN1 expression $(n=4)$. $\mathbf{d}$, e Immunoblotting corresponded with the EN1 expression in qRT-PCR $(n=3)$. $\mathbf{f}, \mathbf{g}$ Nucleus localization of $\beta$-catenin was induced by Wnt activation, but not affected by FGF. White arrows indicate $\beta$-catenin in nucleus (scale bar: $20 \mu \mathrm{m}, n=3$ ). Statistical significance was examined by one-way ANOVA; ${ }^{+\dagger} p=0.001,{ }^{*} p<0.05$

while significantly decreasing the proportion of Glupositive or GABA-positive cells $(4.8 \pm 3.0 \%$ and $2.9 \pm 0.8 \%$, respectively) (Fig. 4d vs. Fig. 4c). Intriguingly, further characterization of the TH-positive cells revealed that they rarely co-expressed LIM homeobox transcription factor 1 alpha (LMX1A), a determinant gene for midbrain DA neurons ${ }^{53}$, or forkhead box protein A2 (FOXA2), a floor plate marker ${ }^{23}$ (Fig. 4e, f, left). This result could be attributed to the fact that authentic midbrain DA neurons originate from the floor plate of the midbrain ${ }^{23}$ and the specification of floor plate fate is only efficient when human PSCs are exposed to an active form of SHH from the early stage of differentiation. Consistently, when we differentiated human ESCs using a previous method based on floor plate differentiation ${ }^{20}$, most of the TH-positive cells strongly co-expressed LMX1A and/or FOXA2 


\section{A}
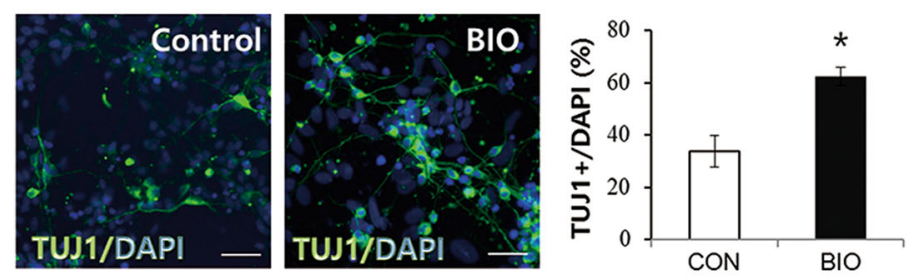

B
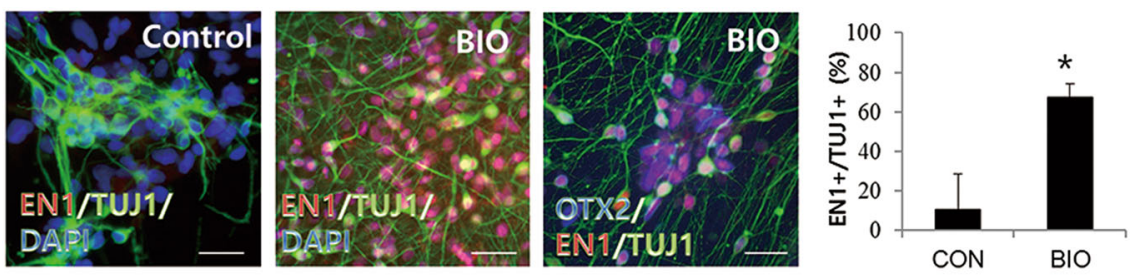

C
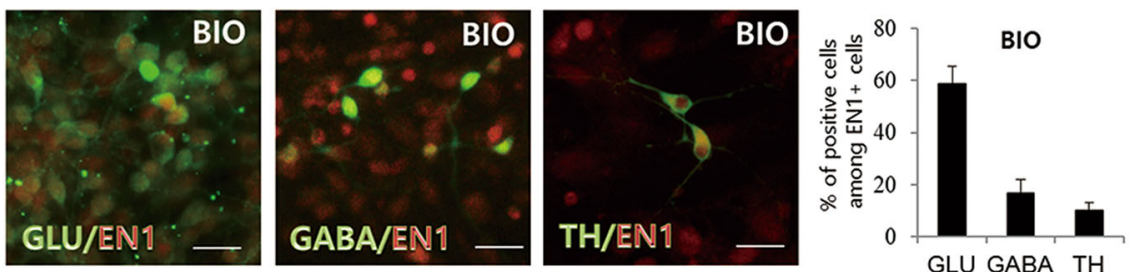

D
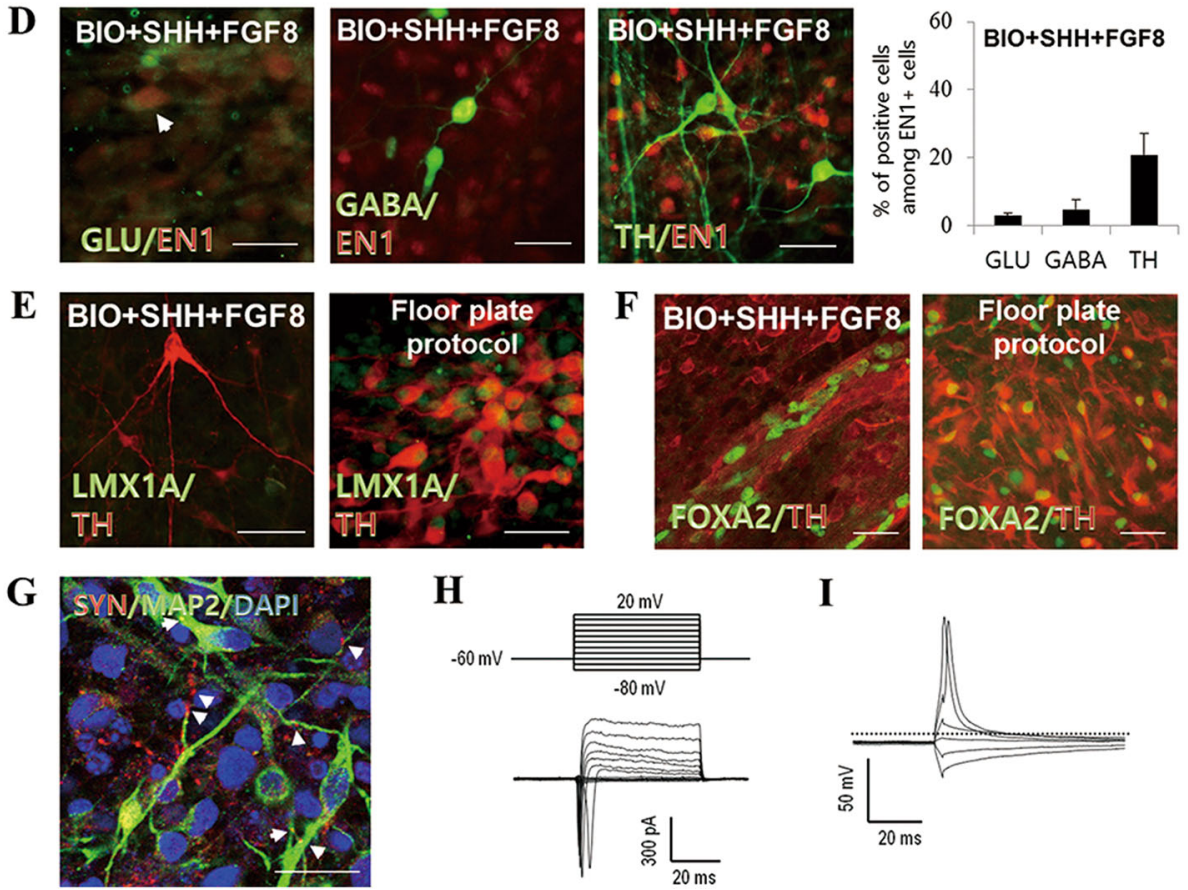

Fig. 4 Generation of functional midbrain neurons from the NPCs pre-exposed to BIO. $\mathbf{a}$, $\mathbf{b}$ NPCs treated with BIO gave rise to TUJ1-positive and TUJ1/EN1 double-positive cells more than non-treated control NPCs. Note that the majority of EN1-positive neurons in BIO-treated group coexpressed OTX2 (b). c NPCs treated with BIO differentiated into various neuronal subtypes, primarily Glu-positive cells followed by GABA-positive and TH-positive cells. $\mathbf{d}$ The proportion of neuronal subtype cells differentiated by treatment with SHH and FGF8 following BIO. TH-positive cells were the most abundant, followed by GABA-positive and Glu-positive cells. e, f TH-positive cells generated by sequential treatment of BIO and SHH + FGF8 rarely co-expressed LMX1A or FOXA2 whereas TH-positive cells differentiated by floor plate-based protocol ${ }^{20}$ were highly positive for both markers. g-i Neurons derived from NPCs treated with BIO were functional, as exemplified by the immunoreactivity of synaptophysin on dendrites (indicated by white arrows; $\mathbf{g}$ ) and voltage-dependent membrane currents (bottom traces; $\mathbf{h}$ ), depolarizing voltage steps (top traces; $\mathbf{h}$ ) as well as evoked action potential by current injection (i). Bars in the graphs are presented as mean \pm standard deviation. Statistical significance was determined by Student's $t$ test; ${ }^{*} p<0.05$. Scale bars: $20 \mu \mathrm{m}$ 
(Fig. 4e, f, right). Thus, few TH-positive cells coexpressing LMX1A or FOXA2 might be resulted from an inefficient ventralization of NPCs because $\mathrm{SHH}$ was treated at a later stage (after BIO treatment) in our differentiation conditions.

Lastly, we assessed the functionality of neurons after the 3-week differentiation. As shown in Fig. 4g, microtubuleassociated protein 2 (MAP2)-positive signals were colocalized with the immunoreactivity of synaptophysin on their dendrites or cell bodies, which indicates that these neurons have the potential to form synapses. We then tested the excitable electrophysiological property of the neurons. The resting membrane potential of the differentiated neurons ranged from -57 to $-65 \mathrm{mV}(-60.8 \pm$ $1.1 \mathrm{mV}, n=8)$. The neurons exhibited voltage-dependent membrane currents that were elicited by $100-\mathrm{ms}$ voltage steps between -80 and $20 \mathrm{mV}$ from a holding potential of $-60 \mathrm{mV}$ (Fig. 4h, $n=8 / 11$ ). Depolarizing voltage steps elicited both large outward potassium currents and fast inward sodium currents. In the current-clamp configuration, a short square current injection ( $3 \mathrm{~ms}$ ) above the supra-threshold induced action potential in the neurons, and prolonged depolarizing current injections $(500 \mathrm{~ms})$ caused the neurons to fire a single action potential (Fig. 4i). These results indicate that the differentiated cells had characteristics of mature neurons.

\section{Discussion}

Wnt family proteins are expressed in the posterior region of vertebrate embryos during gastrulation, and accumulating evidence suggests Wnt signal as a specifying agent in caudal neural characterization ${ }^{11,13}$. Previously, an elegant study provided direct evidence that Wnt signal, in combination with FGF signal, is responsible for inducing posterior characteristics in the neural cells of gastrulating chick embryos in a dose-dependent manner ${ }^{11}$. A later report by the same group showed that cells with the molecular and functional characteristics of the isthmic organizer could be induced from naïve neural plate cells by a combination of Wnt and $\mathrm{FGF}^{54}$.

In substantial agreement with findings from animal models, our data demonstrate that the activation of the canonical Wnt pathway by small-molecule GSK3 inhibitors induces a midbrain-like gene expression profile in human ESC-derived $\mathrm{NPCs}^{49}$. Our data also show that inhibition of the canonical Wnt pathway by either a Wnt signal antagonist or $\beta$-catenin knockdown significantly reduces endogenous $E N 1$ expression, supporting that the canonical Wnt pathway is critical for the midbrain specification of human ESC-derived NPCs. Our data also recapitulate previous results from embryos of lower vertebrates with human ESC-derived NPCs by showing that (1) FGF signal is required for posteriorization by activation of Wnt signal in human ESC-derived $\mathrm{NPCs}^{55}$
(Fig. 3c-e), and that (2) the activation of canonical Wnt signal by GSK3 inhibition elevates the expression of FGF $8^{11,56}$ (Fig. 3a). These findings suggest that both signals cooperate in promoting midbrain-specific gene expression in human ESC-derived NPCs ${ }^{11,55,56}$ and that this cellular machinery is evolutionarily well preserved. Although the detailed molecular mechanism of how Wnt and FGF signals interact to induce EN1 expression in human ESC-derived NPCs requires further study, our data clearly show that FGFs are necessary for induction of $\mathrm{EN1}$, at least in a manner independent of the nuclear translocation of $\beta$-catenin (Fig. 3f, g). Moreover, our work provides molecular evidence that the $\beta$-catenin/TCF complex directly interacts with regulatory element of the EN1 gene in human ESC-derived NPCs. Most importantly, we identified a potential $\beta$-catenin/TCF complexbinding site in the regulatory element of human EN1 gene. As hypothesized, GSK3 inhibition resulted the enhanced binding of the $\beta$-catenin/TCF complex to the putative sites, by which canonical Wnt signal can directly increase the transcriptional level of EN1.

According to the recent studies from two independent groups, activating Wnt signal by applying a different GSK3 inhibitor, CHIR99021, during the neural differentiation of human ESCs induces NPCs to a posterior fate in a dose-dependent manner, and the strong activation of Wnt signal even drives cells to a hindbrain fate ${ }^{21,22}$. In our experiment, however, Wnt signal activation by treatment with BIO (as well as with 1-AKP and lithium) only increased the midbrain specific genes but did not induce the hindbrain genes, even with higher dosage of $\mathrm{BIO}(>1$ $\mu \mathrm{M})$. One possible explanation for this discrepancy would be the usage of the cells at different stages. Unlike the previous studies in which Wnt activation started at the initial stage of neural differentiation (day $0 \sim 3$ ), we examined the effect of Wnt activation with the neural rosette cells, which is a much later stage than in previous studies. As other studies suggest, NPCs in different developmental stages exhibit variable responsiveness to regional patterning cues ${ }^{57,58}$. Therefore, different temporal windows for patterning as well as specific dosages of the patterning cue may result in distinctive regional fates. It is likely that applying patterning cues during early stages of differentiation may be a better strategy if the purpose is purely obtaining neural cells with a particular fate at high efficiency.

It is interesting to note that NPCs treated with BIO predominantly generated glutamatergic and GABAergic neurons (Fig. 4c). In the mouse midbrain, glutamatergic and GABAergic neurons are interspersed in the dorsoventral axis from the inferior and superior colliculi of the dorsal midbrain to the ventral midbrain including the substantia nigra and ventral tegmental area ${ }^{52,59}$. Both subtypes found in various locations of the midbrain are 
highly heterogeneous in morphological and neurochemical properties, and they participate in a variety of neural pathways including relaying somatosensory information and modulating voluntary movement ${ }^{3}$. Even though the distribution of midbrain glutamatergic and GABAergic neurons and the underlying molecular regulation in fate determination of both types have begun to be understood from mouse genetic studies, understanding the functional diversity and detailed differentiation mechanisms of human midbrain neurons is still on high demand owing to their implications in various neurological and psychological diseases ${ }^{52,59,60}$. In this regard, our differentiation paradigm may provide an efficient system for generating a midbrain neuron model that can be used to study developmental mechanisms and roles in relevant neurological disorders.

In conclusion, we demonstrated that the canonical Wnt signal pathway regulates AP patterning during neural differentiation in human PSCs. We show that activating Wnt signal in human ESC-derived NPCs causes neural cells to exhibit midbrain characteristics. More importantly, our data demonstrate that Wnt signal regulates EN1 transcription through direct binding of the $\beta$-catenin/TCF4 complex to the regulatory element of EN1 promoter. Collectively, our finding provides mechanistic insights and technical advances in midbrain specification from human PSCs.

\section{Acknowledgements}

This research was supported by the Bio \& Medical Technology Development Program of the National Research Foundation (NRF) (2017M3A9B4042580), the Basic Science Research Program of the NRF (2015R1D1A1A01056649) from the Ministry of Science and ICT, the Korea Health Technology R\&D Project from the Ministry of Health \& Welfare (HI15C0916), and Korea University Grants (K1504121 and K1505391).

\section{Author details \\ 'Department of Physiology, Yonsei University College of Medicine, 50-1 Yonsei-ro Seodaemun-gu, Seoul 03722, Korea. ${ }^{2}$ Brain Korea 21 PLUS Program for Medical Science, Yonsei University College of Medicine, 50-1 Yonsei-ro, Seodaemun-gu, Seoul 03722, Korea. ${ }^{3}$ Department of Physiology, College of Medicine, Hanyang University, 222 Wangsimni-ro, Seoul 04763, Korea. ${ }^{4}$ Department of Biotechnology, Brain Korea 21 PLUS program for Biotechnology, College of Life Science \& Biotechnology, Korea University, 145 Anam-ro, Seongbuk-gu, Seoul 02841, Korea \\ Author contributions \\ J.Y.K.: Data design, collection and/or assembly, analysis, and interpretation, manuscript writing. J.S.L.: Data collection and/or assembly, analysis, and interpretation. H.S.H., D.R.L., and C.-Y.P.: Data analysis and interpretation. S.J.J., Y. R.Y.: Data collection and/or assembly, analysis. D.-S.K.: Conception and design, data analysis and interpretation, financial support, manuscript writing. D.-W.K.: Conception and design, financial support, data analysis and interpretation, manuscript writing, final manuscript approval.}

\section{Conflict of interest}

The authors declare that they have no conflict of interest.

\section{Publisher's note}

Springer Nature remains neutral with regard to jurisdictional claims in published maps and institutional affiliations.
Supplementary information accompanies this paper at https://doi.org/ 10.1038/s12276-018-0044-y.

Received: 13 September 2017 Revised: 30 November 2017 Accepted: 19 December 2017.

Published online: 13 April 2018

\section{References}

1. Bissonette, G. B. \& Roesch, M. R. Development and function of the midbrain dopamine system: what we know and what we need to. Genes Brain Behav. 15, 62-73 (2006).

2. Morello, F. \& Partanen, J. Diversity and development of local inhibitory and excitatory neurons associated with dopaminergic nuclei. FEBS Lett. 589, 3693-3701 (2015).

3. Stein, B. E., Stanford, T. R. \& Rowland, B. A. Development of multisensory integration from the perspective of the individual neuron. Nat. Rev. Neurosci. 15, 520-535 (2014).

4. Hutchinson, M. et al. Cervical dystonia: a disorder of the midbrain network for covert attentional orienting. Front. Neurol. 5, 54 (2014).

5. Volkow, N. D. \& Morales, M. The brain on drugs: from reward to addiction. Cell 162, 712-25 (2015).

6. Chen, M. C., Yu, H., Huang, Z. L. \& Lu, J. Rapid eye movement sleep behavior disorder. Curr. Opin. Neurobiol. 23, 793-798 (2013).

7. Blaess, S. \& Ang, S. L. Genetic control of midbrain dopaminergic neuron development. Wiley Interdiscip. Rev. Dev. Biol. 4, 113-34 (2015).

8. Arenas, E., Denham, M. \& Villaescusa, J. C. How to make a midbrain dopaminergic neuron. Development 14, 1918-1936 (2015).

9. Castelo-Branco, G. \& Arenas, E. Function of Wnts in dopaminergic neuron development. Neurodegener. Dis. 3, 5-11 (2006).

10. Alves dos Santos, M. T. \& Smidt, M. P. En1 and Wnt signaling in midbrain dopaminergic neuronal development. Neural Dev. 6, 23 (2011).

11. Nordström, U., Jessell, T. M. \& Edlund, T. Progressive induction of caudal neural character by graded Wnt signaling. Nat. Neurosci. 5, 525-532 (2002).

12. McMahon, A. P. \& Bradley, A. The Wnt-1 (int-1) proto-oncogene is required for development of a large region of the mouse brain. Cell 62, 1073-1085 (1990).

13. Thomas, K. R. \& Capecchi, M. R. Targeted disruption of the murine int-1 protooncogene resulting in severe abnormalities in midbrain and cerebellar development. Nature 346, 847-850 (1990).

14. Danielian, P. S. \& McMahon, A. P. Engrailed-1 as a target of the Wnt-1 signalling pathway in vertebrate midbrain development. Nature 383, 332-334 (1996).

15. Glinka, A. et al. Dickkopf-1 is a member of a new family of secreted proteins and functions in head induction. Nature 391, 357-362 (1998).

16. Piccolo, S. et al. The head inducer Cerberus is a multifunctional antagonist of Nodal, BMP and Wnt signals. Nature 397, 707-710 (1999).

17. Wurst, W., Auerbach, A. B. \& Joyner, A. L. Multiple developmental defects in Engrailed-1 mutant mice: an early mid-hindbrain deletion and patterning defects in forelimbs and sternum. Development 120, 2065-2075 (1994).

18. Wassarman, K. M. et al. Specification of the anterior hindbrain and establishment of a normal mid/hindbrain organizer is dependent on Gbx2 gene function. Development 124, 2923-2934 (1997).

19. Moya, N., Cutts, J., Gaasterland, T., Willert, K. \& Brafman, D. A. Endogenous WNT signaling regulates hPSC-derived neural progenitor cell heterogeneity and specifies their regional identity. Stem Cell Rep. 3, 1015-1028 (2014).

20. Kriks, $\mathrm{S}$. et al. Dopamine neurons derived from human ES cells efficiently engraft in animal models of Parkinson's disease. Nature 480, 547-551 (2011).

21. Xi, J., Liu, Y., Liu, H., Chen, H., Emborg, M. E. \& Zhang, S. C. Specification of midbrain dopamine neurons from primate pluripotent stem cells. Stem Cells 30, 1655-1663 (2012).

22. Kirkeby, A. et al. Generation of regionally specified neural progenitors and functional neurons from human embryonic stem cells under defined conditions. Cell Rep. 1, 703-714 (2012).

23. Joksimovic, M. et al. Wht antagonism of Shh facilitates midbrain floor plate neurogenesis. Nat. Neurosci. 12, 125-31 (2009).

24. Fasano, C. A., Chambers, S. M., Lee, G., Tomishima, M. J. \& Studer, L. Efficient derivation of functional floor plate tissue from human embryonic stem cells. Cell Stem Cell 6, 336-47 (2010)

25. Partanen, J. FGF signalling pathways in development of the midbrain and anterior hindbrain. J. Neurochem. 101, 1185-1193 (2007). 
26. Lahti, L., Peltopuro, P., Piepponen, T. P. \& Partanen, J. Cell-autonomous FGF signaling regulates anteroposterior patterning and neuronal differentiation in the mesodiencephalic dopaminergic progenitor domain. Development 139 , 894-905 (2012).

27. Ye, W., Shimamura, K., Rubenstein, J. L., Hynes, M. A. \& Rosenthal, A. FGF and Shh signals control dopaminergic and serotonergic cell fate in the anterior neural plate. Cell 93, 755-766 (1998).

28. Yan, Y. et al. Directed differentiation of dopaminergic neuronal subtypes from human embryonic stem cells. Stem Cells 23, 781-790 (2005).

29. Jang, J. et al. Disease-specific induced pluripotent stem cells: a platform for human disease modeling and drug discovery. Exp. Mol. Med. 44, 202-213 (2012).

30. Park, C. Y. et al. Targeted inversion and reversion of the blood coagulation factor 8 gene in human iPS cells using TALENs. Proc. Natl Acad. Sci. USA 111, 9253-9258 (2014).

31. Kim, D. S. et al. Robust enhancement of neural differentiation from human ES and iPS cells regardless of their innate difference in differentiation propensity. Stem Cell Rev. 6, 270-281 (2010).

32. Elkabetz, Y. et al. Human ES cell-derived neural rosettes reveal a functionally distinct early neural stem cell stage. Genes Dev. 22, 152-165 (2008).

33. Gaspard, N. \& Vanderhaeghen, P. Mechanisms of neural specification from embryonic stem cells. Curr. Opin. Neurobiol. 20, 37-43 (2010).

34. Meijer, L. et al. GSK-3-selective inhibitors derived from Tyrian purple indirubins. Chem. Biol. 10, 1255-1266 (2003).

35. Sato, N., Meijer, L., Skaltsounis, L., Greengard, P. \& Brivanlou, A. H. Maintenance of pluripotency in human and mouse embryonic stem cells through activation of Wnt signaling by a pharmacological GSK-3-specific inhibitor. Nat. Med. 10, 55-63 (2004).

36. Murphy, D. B. et al. Human brain factor 1, a new member of the fork head gene family. Genomics 21, 551-557 (1994).

37. Lagutin, O. V. et al. Six3 repression of Wnt signaling in the anterior neuroectoderm is essential for vertebrate forebrain development. Genes Dev. 17, 368-379 (2003).

38. Pfeffer, P. L., Payer, B., Reim, G., di Magliano, M. P. \& Busslinger, M. The activation and maintenance of Pax2 expression at the mid-hindbrain boundary is controlled by separate enhancers. Development 129, 307-318 (2002).

39. Prince, V. E., Moens, C. B., Kimmel, C. B. \& Ho, R. K. Zebrafish hox genes: expression in the hindbrain region of wild-type and mutants of the segmentation gene, valentino. Development 125, 393-406 (1998).

40. Kunick, C., Lauenroth, K., Leost, M., Meijer, L. \& Lemcke, T. 1-Azakenpaullone is a selective inhibitor of glycogen synthase kinase-3 beta. Bioorg. Med. Chem. Lett. 14, 413-416 (2004).

41. Phiel, C. J. \& Klein, P. S. Molecular targets of lithium action. Annu. Rev. Phamacol. Toxicol. 41, 789-813 (2011).

42. Zhang, F., Phiel, C. J., Spece, L., Gurvich, N. \& Klein, P. S. Inhibitory phosphorylation of glycogen synthase kinase-3 (GSK-3) in response to lithium. Evidence for autoregulation of GSK-3. J. Biol. Chem. 278, 33067-33077 (2003).
43. MacDonald, B. T., Tamai, K. \& He, X. Wnt/beta-catenin signaling: components, mechanisms, and diseases. Dev. Cell 17, 9-26 (2009).

44. Rao, T. P. \& Küh, M. An updated overview on Wht signaling pathways: a prelude for more. Circ. Res. 106, 1798-806 (2010).

45. McMahon, A. P., Joyner, A. L., Bradley, A. \& McMahon, J. A. The midbrainhindbrain phenotype of Wnt-1-Nnt-1- mice results from stepwise deletion of engrailed-expressing cells by 9.5 days postcoitum. Cell 69, 581-595 (1992).

46. McGrew, L. L., Otte, A. P. \& Moon, R. T. Analysis of Xwnt-4 in embryos of Xenopus laevis: a Wnt family member expressed in the brain and floor plate. Development 115, 463-473 (1992).

47. Kiecker, C. \& Niehrs, C. A morphogen gradient of Wnt/beta-catenin signalling regulates anteroposterior neural patterning in Xenopus. Development $\mathbf{1 2 8}$, 4189-4201 (2001).

48. Muhr, J., Graziano, E., Wilson, S., Jessell, T. M. \& Edlund, T. Convergent inductive signals specify midbrain, hindbrain, and spinal cord identity in gastrula stage chick embryos. Neuron 23, 689-702 (1999).

49. Liu, A. \& Joyner, A. L. Early anterior/posterior patterning of the midbrain and cerebellum. Annu. Rev. Neurosci. 24, 869-896 (2001).

50. Krejci, P. et al. Receptor tyrosine kinases activate canonical WNT/ß-catenin signaling via MAP kinase/LRP6 pathway and direct $\beta$-catenin phosphorylation. PLOS ONE 7, e35826 (2012).

51. Joksimovic, M. \& Awatramani, R. Wnt/ß-catenin signaling in midbrain dopaminergic neuron specification and neurogenesis. J. Mol. Cell Biol. 6, 27-33 (2014).

52. Lahti, L., Achim, K. \& Partanen, J. Molecular regulation of GABAergic neuron differentiation and diversity in the developing midbrain. Acta Physiol. 207 616-627 (2013).

53. Andersson, E. et al. Identification of intrinsic determinants of midbrain dopamine neurons. Cell 124, 393-405 (2006).

54. Olander, S., Nordström, U., Patthey, C. \& Edlund, T. Convergent Wnt and FGF signaling at the gastrula stage induce the formation of the isthmic organizer. Mech. Dev. 123, 166-176 (2006).

55. Domingos, P. M. et al. The Wnt/beta-catenin pathway posteriorizes neural tissue in Xenopus by an indirect mechanism requiring FGF signalling. Dev. Biol. 239, 148-160 (2001).

56. Canning, C. A., Lee, L., Irving, C., Mason, I. \& Jones, C. M. Sustained interactive Wnt and FGF signaling is required to maintain isthmic identity. Dev. Biol. $\mathbf{3 0 5}$ 276-286 (2007).

57. Li, X. J. et al. Specification of motor neurons from human embryonic stem cells. Nat. Biotechnol. 23, 215-221 (2005).

58. Zhang, S. C. Neural subtype specification from embryonic stem cells. Brain Pathol. 16, 132-142 (2006).

59. Morales, M. \& Root, D. H. Glutamate neurons within the midbrain dopamine regions. Neuroscience 282, 60-68 (2014).

60. Doherty, D., Millen, K. J. \& Barkovich, A. J. Midbrain and hindbrain malformations: advances in clinical diagnosis, imaging, and genetics. Lancet Neurol. 12, 381-393 (2013). 